\title{
COVID-19 grave y embarazo. A propósito de dos casos alrededor de la viabilidad fetal
}

\section{Severe COVID-19 and pregnancy. On purpose of 2 cases around fetal viability}

\author{
Ulises Guajardo1,2*, Horacio Figueroa ${ }^{1,2}$, Arturo Soldati ${ }^{1,2}$, Juan Baltar ${ }^{3}$, Carlos Rivera ${ }^{4}$ y \\ Enrique Oyarzún $n^{1,2}$
}

${ }^{1}$ Servicio de Obstetricia y Ginecología, Clínica Universidad de los Andes; ${ }^{2}$ Departamento de Obstetricia, Ginecología y Biología de la Reproducción, Universidad de los Andes; ${ }^{3}$ Unidad de Paciente Crítico, Clínica Universidad de los Andes; ${ }^{4}$ Servicio de Urgencia, Clínica Universidad de los Andes. Santiago, Chile

\section{Resumen}

El presente trabajo describe la evolución de dos casos clínicos graves de COVID-19 en pacientes embarazadas con 27 y 24 semanas de amenorrea. A partir de estos casos se resume la evidencia disponible en la literatura en relación con el curso grave de la enfermedad durante el embarazo y se sugieren guías para considerar en la reflexión multidisciplinaria que permite manejar y resolver casos similares.

Palabras clave: COVID-19. SARS-CoV-2. Embarazo.

\section{Abstract}

The present article describes the evolution of two clinical cases of severe COVID-19 in pregnant patients with 27 and 24 weeks of gestational weeks. The available up-to-date evidence about severe course of the disease during pregnancy is resumed. Management guides are suggested for the multidisciplinary approach of similar cases.

Key words: COVID-19. SARS-CoV-2. Pregnancy.

\section{Introducción}

En diciembre de 2019, el gobierno chino reportó un brote poblacional de neumonía por un nuevo beta-coronavirus que fue denominado Severe Acute Respiratory Syndrome Coronavirus 2 (SARS-CoV-2). La enfermedad causada por este virus se conoce como COVID-19 (Coronavirus Disease 2019). En marzo de 2020, la Organización Mundial de la Salud declaró que la propagación de la enfermedad se había constituido en pandemia. Muchos aspectos de la COVID-19 son aún desconocidos y determinan un desafío para los equipos de salud y los servicios sanitarios ${ }^{1}$.

Las mujeres embarazadas no están exentas de esos desafíos. Por una parte, siempre se ha considerado a las gestantes más susceptibles de enfermar y de comprometer al feto in utero, y por otra parte, los coronavirus previos, al igual que la influenza, afectaron más
Disponible en internet: 02-08-2021

\section{Rev Chil Obstet Ginecol. 2021;86(3):309-316
www. rechog.com \\ Rev Chil Obstet Ginecol. 2021;86(3):309-316
www. rechog.com} 0048-766X / ๑ 2021 Sociedad Chilena de Obstetricia y Ginecología. Publicado por Permanyer. Éste es un artículo open access bajo la licencia CC BY-NC-ND (https://creativecommons.org/licenses/by-nc-nd/4.0/). 
gravemente a las embarazadas que a las mujeres no embarazadas ${ }^{2}$.

Los resultados reportados hasta ahora en cinco revisiones sistemáticas y una cohorte prospectiva poblacional señalan que la COVID-19 se presenta entre las 34 y 36 semanas de gestación en promedio, y que los síntomas más frecuentes son tos y fiebre. Los hallazgos de laboratorio más habituales son linfocitopenia, trombocitopenia y elevación del dímero $\mathrm{D}$, la proteína $\mathrm{C}$ reactiva y las transaminasas. Desde el punto de vista perinatal, en la revisión sistemática con mayor número de casos de embarazadas con COVID-19 $(n=538)$, la frecuencia de parto prematuro fue del $20,1 \%$, con una tasa de cesárea del $84,7 \%{ }^{3}$.

A pesar de que el SARS-CoV-2 comparte el $85 \%$ de su material genético con el SARS-CoV de 2003, no se ha comportado de la misma forma, pues presenta menor gravedad en la población femenina, gestante o no gestante ${ }^{4}$. Sin embargo, en relación al embarazo existe un problema adicional, como es la presencia de la enfermedad en su forma grave en el contexto de una gestación en el límite de la viabilidad fetal.

\section{Caso clínico 1}

Paciente de 43 años, multípara de tres partos vaginales, cursando embarazo de $27+3$ semanas, con antecedentes de diabetes mellitus tipo 2 requirente de insulina, mal control metabólico y contacto estrecho con paciente COVID-19 positivo. Consultó a urgencia por un cuadro de 3 días de evolución con compromiso del estado general, vómitos, disnea progresiva, odinofagia, malestar torácico y dinámica uterina. Al ingreso se encontraba hipertensa, taquicárdica, taquipneica y afebril, y presentaba un examen pulmonar alterado con radiografía de tórax compatible con neumonía multifocal. La evaluación obstétrica certificó vitalidad fetal y ausencia de dinámica uterina y de modificaciones cervicales. Fue ingresada a la unidad de cuidados intensivos (UCI) con diagnóstico de neumonía (probable COVID-19) y cetoacidosis diabética grave. Al laboratorio destacaban linfocitopenia, proteína $C$ reactiva elevada y dímero D elevado (> $2000 \mathrm{ng} / \mathrm{ml}$ ). En la evaluación ecográfica se evidenció un feto con crecimiento adecuado, polihidramnios y perfil biofísico 8/8. Se inició terapia antibiótica de amplio espectro y tromboprofilaxis. Además, recibió corticoides para la inducción de la maduración pulmonar fetal. Al tercer día de hospitalización evolucionó hipertensa, con edema pulmonar agudo y cetoacidosis de difícil manejo, por lo que se decidió la interrupción del embarazo vía cesárea, previa intubación y ventilación mecánica invasiva (VMI) luego de 2 horas de oxigenoterapia por cánula de alto flujo sin mejoría. Al quinto día de hospitalización se notificó reacción en cadena de la polimerasa (PCR) en tiempo real positiva para SARS-CoV-2. Se mantuvo en VMI por 5 días, con mejoría de los parámetros respiratorios, y requirió apoyo transfusional. Fue dada de alta tras 15 días de hospitalización en buenas condiciones. El recién nacido pesó 880 gramos, obtuvo Apgar 6 y 8 al minuto y los 5 minutos de vida, respectivamente, y requirió intubación, ventilación mecánica y estadía de 41 días en la unidad de cuidados intensivos neonatales. Se realizó búsqueda activa de SARS-CoV-2 con dos PCR en hisopado nasofaríngeo antes de las 12 horas de vida, ambos con resultado negativo.

\section{Caso clínico 2}

Paciente obesa, de 33 años, paridad de 1, cursando embarazo de $24+1$ semanas. Consulta en urgencia por un cuadro de 9 días de evolución caracterizado por compromiso del estado general, fiebre, tos y congestión nasal, a lo que se agrega disnea los 2 días anteriores a la consulta. Tenía PCR positiva para SARS-CoV-2 solicitada de forma ambulatoria al inicio de los síntomas. En la exploración destacaban taquicardia, taquipnea y fiebre. En la evaluación obstétrica se certificó vitalidad fetal y crecimiento acorde con la edad gestacional. En la tomografía computarizada de tórax se evidenció neumonía multifocal. Fue ingresada en la $\mathrm{UCl}$ con los diagnósticos de insuficiencia respiratoria y neumonía por COVID-19. Recibió terapia antibiótica de amplio espectro y al tercer día de hospitalización se decidió intubación endotraqueal para VMI por síndrome de dificultad respiratoria grave, tras 2 días de oxigenoterapia por cánula de alto flujo sin mejoría. Además, recibió heparina de bajo peso molecular en dosis terapéuticas y corticoterapia. Una ecotomografía abdominal solicitada por fiebre persistente sin foco claro mostró hepatomegalia, esteatosis difusa, colelitiasis y pólipos vesiculares. Posteriormente cursó con falla renal aguda de origen mixto, con proteinuria persistente, actualmente en resolución. Además, presentó hipertrigliceridemia y pancreatitis aguda multifactorial. Fue extubada con éxito tras 18 días de VMI, con dos periodos de pronación incluidos. Posteriormente ha presentado cifras de presión arterial elevadas ocasionales, sin crisis hipertensivas y sin diagnóstico de preeclampsia. Al momento de escribir este artículo se encuentra en seguimiento neurológico 
por electroencefalograma alterado en contexto de delirium hiperactivo posextubación (neuroimágenes sin hallazgos patológicos) y cursa embarazo de $27+3$ semanas, sin requerimientos de oxígeno suplementario y con evaluación obstétrica diaria que demuestra vitalidad y bienestar fetal.

\section{Discusión}

La evidencia sobre el curso grave de la enfermedad en pacientes embarazadas aún es escasa, pero permite hacer algunas consideraciones sobre su manejo. Según diversos reportes, la proporción de embarazadas que requerirán hospitalización en la $\mathrm{UCl}$ por COVID-19 es baja $(0,7-2,8 \%)$, y similar a la que requerirá VMI $(0,5-1,7 \%)^{5,6}$.

La experiencia francesa acumulada entre marzo y abril de 2020 (35 casos graves entre 617 embarazadas de 33 maternidades) indicó que la gravedad de la infección durante el embarazo se asoció a una edad materna mayor de 35 años, obesidad, diabetes preexistente e hipertensión arterial en cualquiera de sus formas. Esta experiencia mostró que en los casos graves o críticos el parto ocurrió antes de las 37 semanas en 23 de 29 pacientes, con una madre fallecida y un recién nacido prematuro que también falleció?.

La asociación de enfermedad grave y morbilidad materna asociada o preexistente también ha sido señalada por las experiencias china, norteamericana y británica publicadas ${ }^{8-11}$. La tasa de parto prematuro antes de las 37 semanas de gestación en la cohorte británica prospectiva del grupo UKOSS (427 pacientes) fue del $28 \%$ en algo más de la mitad de las mujeres que habían resuelto su parto para el momento del análisis. En esta cohorte, la tasa de mortalidad materna fue de 5,8 $(1,9-13,5)$ por cada 100.000 embarazos ${ }^{11}$.

Los Centers for Disease Control and Prevention de los Estados Unidos de América han publicado un reporte sobre COVID-19 y mujeres en edad reproductiva. La publicación da cuenta de 326.335 mujeres entre 15 y 44 años con COVID-19 confirmada entre enero y junio de 2020, de las cuales 8297 estaban embarazadas. Entre las mujeres embarazadas fue más frecuente la presencia de diabetes, enfermedades cardiovasculares y enfermedades pulmonares crónicas. El riesgo de hospitalización fue mayor en las embarazadas que en las no embarazadas, pero el riesgo de muerte fue similar en ambos grupos. Además, el reporte destaca el hecho de que las mujeres hispanas y afrodescendientes fueron más afectadas durante la gestación ${ }^{12}$.

\section{Consideraciones respecto a la hospitalización de embarazadas con COVID-19}

Debe considerarse la hospitalización en todas las pacientes embarazadas que consultan en urgencia con sospecha de COVID-19 y síntomas o signos moderados a graves, como disnea, taquipnea, taquicardia, fiebre o saturación capilar de oxígeno $<95 \%{ }^{13}$. También deben ser hospitalizadas las pacientes con factores que aumenten el riesgo de un curso más grave de la enfermedad ${ }^{14}$.

Las pacientes que requieren hospitalización deben ser aisladas y monitorizadas de forma estricta durante las primeras $24-48$ horas. Si bien se solicitan sistemáticamente pruebas de laboratorio e imágenes básicas, en las pacientes con hipoxemia mantenida se debe ampliar el estudio buscando diagnosticar insuficiencia respiratoria de forma precoz y descartar otras causas $^{15}$. La indicación de oxigenoterapia debe ser temprana, buscando saturaciones sobre el $96 \%^{16}$, y debe considerarse la utilización de la posición de prono o semiprono vigil ${ }^{17,18}$ (Fig. 1), cuya utilidad y seguridad también están demostradas durante la gestación ${ }^{19}$. Si no se logra el objetivo en un tiempo acotado, se debe escalar al uso de cánula de alto flujo y luego a intubación endotraqueal y VMI. Se desaconseja el uso de sistemas de ventilación mecánica no invasiva por el riesgo de generación de aerosoles ${ }^{20}$. El uso de VMI ha sido uno de los pilares en el manejo de la COVID19 grave, tanto en mujeres no embarazadas como embarazadas ${ }^{21}$.

En el contexto del embarazo, debe considerarse que la interrupción de este no mejora necesariamente la condición respiratoria de la paciente ${ }^{22}$, si bien, según sea la edad gestacional, puede resultar conveniente la interrupción del embarazo en pacientes particularmente graves.

\section{Consideraciones respecto al uso de corticoides}

Los resultados preliminares del ensayo clínico RECoVERY han demostrado que el uso de dexametasona en dosis de $6 \mathrm{mg}$ diarios por hasta 10 días reduce la mortalidad de manera significativa en los pacientes con COVID-19 que requieren oxigenoterapia (21.5\% frente a $25 \%)$ o VMI $(29 \% \text { frente a } 40.7 \%)^{23,24}$. En este 


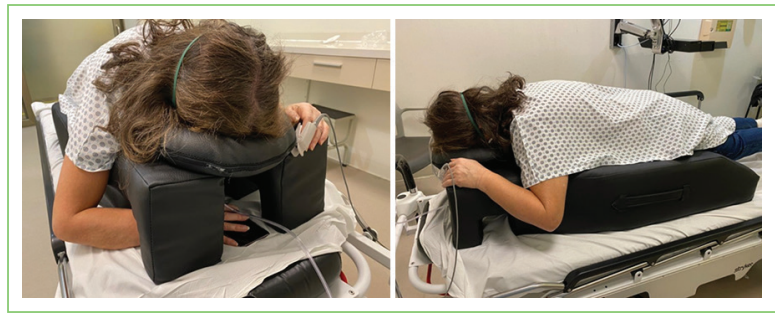

Figura 1. Colchón para pronación vigil utilizado en la Clínica Universidad de los Andes, adaptado del original del Dr. Richard Levita. El colchón mejora la comodidad de la paciente y permite acojinar las zonas de presión. (Cortesía del Dr. Cristián Garrido, con autorización de la paciente.)

contexto no debe restringirse el uso de corticoides en embarazos pretérmino; por el contrario, debe recordarse que, en relación al uso de corticoides para la prevención de los riesgos posnatales asociados a la prematuridad, la dexametasona está avalada a partir de su utilización en el estudio del grupo colaborativo estadounidense en terapia esteroidal antenatal ${ }^{25}$, uno de los siete ensayos clínicos con dexametasona que la base de datos Cochrane resume para tales efectos ${ }^{26}$.

\section{Consideraciones respecto al uso de tratamiento anticoagulante}

Se ha reportado una incidencia aumentada de eventos tromboembólicos en el curso grave de la COVID19, incluyendo pacientes embarazadas ${ }^{27}$. En un reporte de 3334 pacientes hospitalizados con diagnóstico de COVID-19 en Nueva York ocurrió un evento trombótico en el $16 \%$, de los cuales el $6,2 \%$ fueron venosos $(3,2 \%$ tromboembolia pulmonar y $3,9 \%$ trombosis venosa profunda) y el $11,1 \%$ arteriales $(1,6 \%$ accidente cerebro vascular $[\mathrm{ACV}]$ isquémico, $8,9 \%$ infarto miocárdico y $1 \%$ embolia sistémica). Interesantemente, los pacientes recibían anticoagulación profiláctica. Los factores de riesgo asociados fueron ser hispano, tener enfermedad coronaria o infarto previo, y sobre todo un valor alto de dímero $\mathrm{D}$, con un incremento significativo del riesgo a partir de los $500 \mathrm{ng} / \mathrm{ml}$, pero en especial por encima de los $2000 \mathrm{ng} /$ $\mathrm{ml}$, tanto para trombosis arterial como para trombosis venosa. Por esta razón, salvo contraindicación formal, está indicada la tromboprofilaxis farmacológica en todos los pacientes con COVID-19 hospitalizados, y anticoagulación plena en los de mayor riesgo, en particular en las pacientes obstétricas ${ }^{28,29}$.
Consideraciones respecto a la evaluación del bienestar fetal

Con respecto a la evaluación del bienestar fetal, se ha propuesto la monitorización intermitente de la actividad cardiaca antes de las 28 semanas y la monitorización fetal continua desde las 28 semanas de gestación en las pacientes graves ${ }^{30}$. Nuestro grupo considera que la monitorización continua debe realizarse desde las 26 semanas en adelante, porque esta es una edad gestacional asociada a un peso cercano a $1000 \mathrm{~g}$, límite para plantearse siempre la posibilidad de interrupción del embarazo en patologías que comprometen la salud materna. En los países con menos recursos e indicadores diferentes, 28 semanas podría reemplazar ese criterio.

En general, en la actualidad se consideran 24 semanas de amenorrea como límite de viabilidad fetal, y se conocen bien los resultados en el seguimiento de los prematuros que nacen a esa edad gestacional. Consideramos, sin embargo, que incluso en pacientes graves el manejo del embarazo entre las 24 y las 26 semanas debe ser conservador, intentando mantener al feto in utero. No creemos que a esa edad gestacional el pronóstico materno cambie con la extracción fetal, y desde el punto de vista de la monitorización fetal creemos que en esos rangos de edad gestacional basta con auscultar latidos cardiacos fetales diariamente para certificar la vitalidad.

Desde las 26 semanas en adelante creemos que lo conveniente y práctico es la monitorización electrónica diaria de los latidos cardiacos fetales, durante 40 minutos 01 hora, buscando desaceleraciones espontáneas o tardías que sugerirían una condición ominosa fetal. A lo anterior agregaríamos la evaluación bisemanal del líquido amniótico y, eventualmente, Doppler de arteria umbilical. Esto siempre en el contexto de una paciente grave en la $\mathrm{UCl}$, con ventilación mecánica, donde la monitorización continua y permanente del feto resulta difícil, si no imposible (mucho más en caso de estar la paciente en prono).

Desde las 26 semanas en adelante también resulta conveniente evaluar el crecimiento fetal con biometría cada 2 semanas, si las condiciones lo permiten.

\section{Consideraciones respecto a la inclusión de embarazadas en estudios de diferentes fármacos para el tratamiento de la COVID-19}

No nos parece prudente incluir embarazadas en los estudios que evalúen fármacos cuyos efectos se 


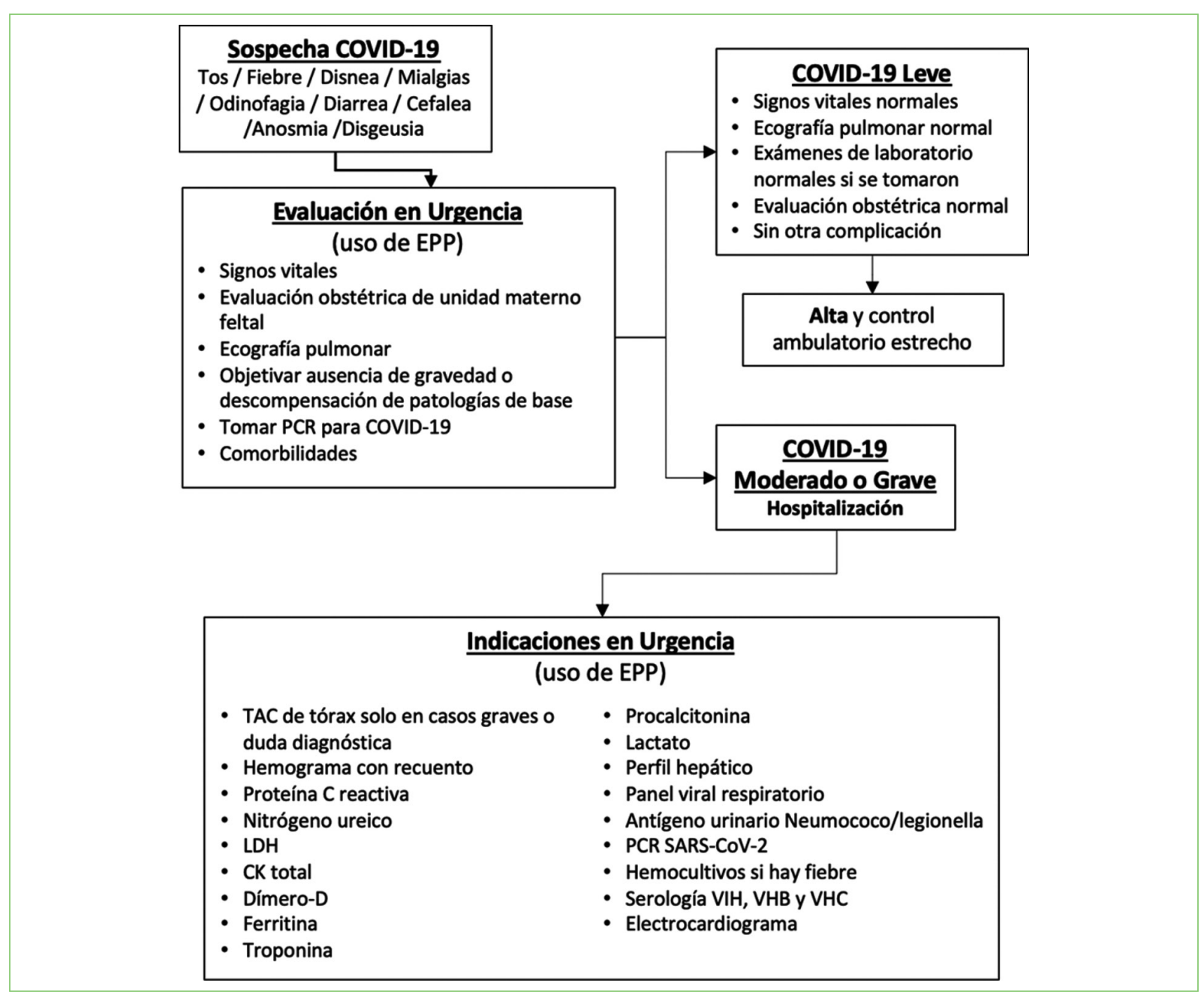

Figura 2. Propuesta de evaluación y criterios de hospitalización de pacientes gestantes con COVID-19 en el Servicio de Urgencia. CK: creatinquinasa; EPP: elementos de protección personal; LDH: deshidrogenasa láctica; PCR: reacción de polimerasa en cadena; TAC: tomografía axial computarizada; VHB: virus hepatitis B; VHC: virus hepatitis $\mathrm{C} ; \mathrm{VIH}$ : virus de inmunodeficiencia humana.

desconocen y respecto de los cuales no hay garantías de mejoría del cuadro de base.

\section{Consideraciones respecto a la interrupción del embarazo}

La indicación de interrupción del embarazo debe guiarse por criterios obstétricos, al igual que la vía de interrupción, considerando un balance entre los riesgos y los beneficios maternos y fetales ${ }^{24}$. La VMI no es contraindicación de parto vaginal $y$, de hecho, ya existe un reporte de inducción con parto instrumental en una paciente intubada ${ }^{31}$.

Creemos que siendo ideal, en general, alcanzar las 32 semanas de edad gestacional para ofrecer un mejor pronóstico al feto y al recién nacido, cuando se trate de decidir la interrupción de la gestación por gravedad materna o por compromiso de la unidad fetoplacentaria lo deseable es que la edad gestacional sea de al menos 26 semanas. La interrupción del embarazo entre las semanas 24 y 26 debe ser una decisión cuidadosamente meditada por el equipo tratante y resuelta caso a caso. No puede haber guías o recetas para esa situación. Por debajo de las 24 semanas, por otra parte, no existe en este contexto justificación para la interrupción del embarazo, porque ello no modificaría ni beneficiaría el manejo materno ni en casos de extrema gravedad, de modo que la situación de COVID-19 y embarazo no sería la misma que podría representar una preeclampsia grave antes de las 24 semanas de gestación (situación en la cual la interrupción del embarazo «mejora» a la madre). 


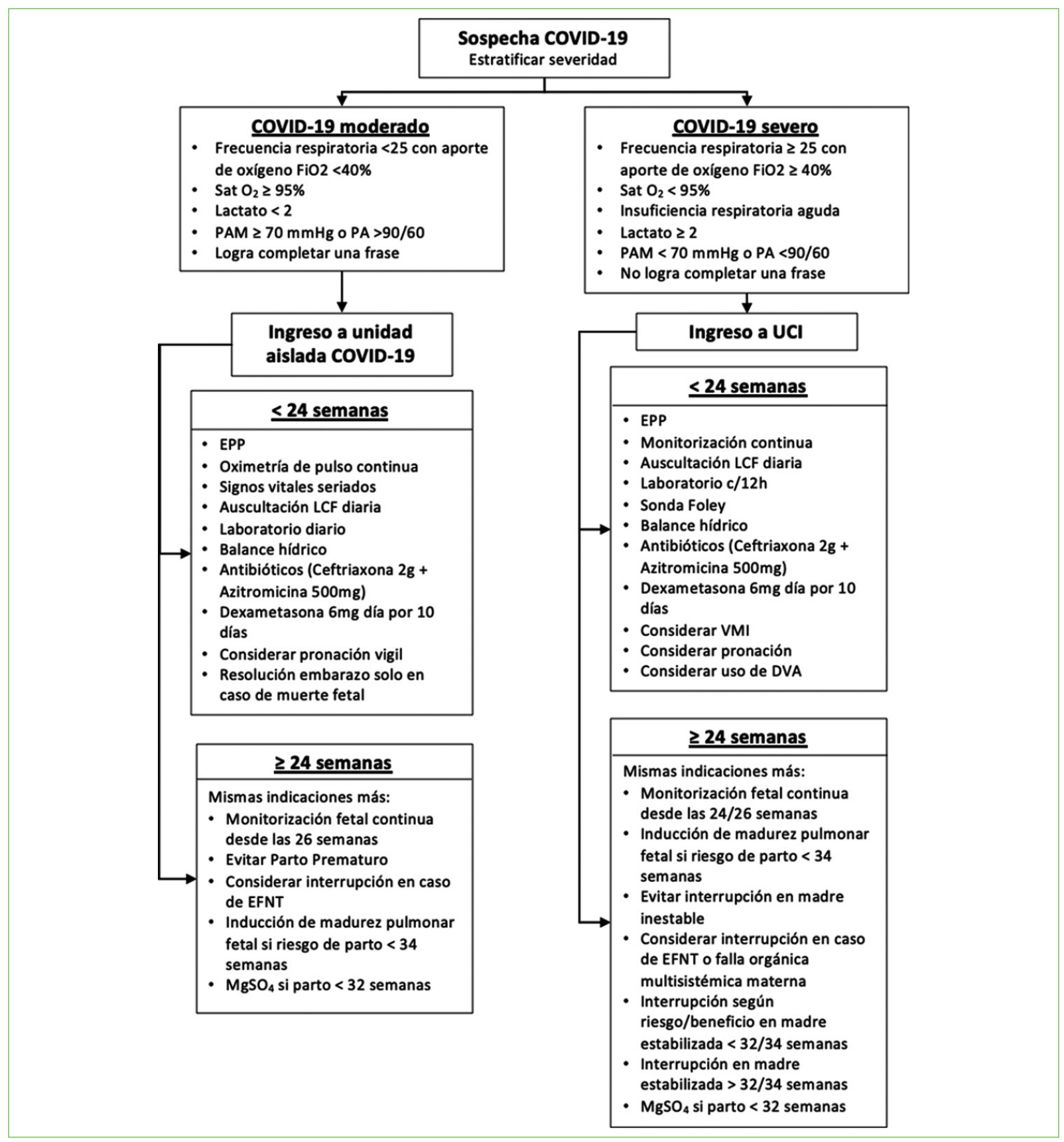

Figura 3. Propuesta de evaluación y manejo de pacientes gestantes con COVID-19 moderado y severo. EFNT: estado fetal no tranquilizador; EPP: elementos de protección personal; LCF: latidos cardíacos fetales; MgS04: sulfato de magnesio; DVA: drogas vasoactivas; PA: presión arterial; PAM: presión arterial media; UCI: unidad de cuidados intensivos; VMI: ventilación mecánica invasiva.

Dado que 32 semanas o $1500 \mathrm{~g}$ de peso fetal estimado se asocian muy significativamente a una disminución de las complicaciones propias de la prematuridad (membrana hialina, hemorragia intracraneana, enterocolitis necrotizante), creemos que esa edad gestacional y ese peso fetal estimado constituyen una indicación de interrupción del embarazo en pacientes graves que van a requerir VMI u otros procedimientos y tratamientos. Esa edad gestacional no solo se asocia a menores riesgos para el feto y el recién nacido, sino también a mayores dificultades de ventilación materna por los cambios inherentes a un volumen uterino mayor en 
caso de no interrumpir el embarazo. Naturalmente, la interrupción del embarazo debe llevarse adelante con la paciente estabilizada, porque lo contrario se asociaría a peores consecuencias para la madre y el recién nacido.

Creemos que la probable discusión en relación a utilizar el criterio de 32 semanas o el de 34 semanas solo puede basarse en la experiencia del equipo tratante frente a una pregunta para la cual no existen estudios aleatorizados que puedan contestarla en mejor forma. En la decisión de interrupción del embarazo debe considerarse, al igual que en el manejo de pacientes embarazadas graves en general, la opinión de todos los miembros del equipo multidisciplinario a cargo de la paciente.

En caso de parto antes de las 32 semanas se debe indicar sulfato de magnesio según las recomendaciones habituales ${ }^{32}$.

\section{Consideraciones respecto a la posibilidad de una cesárea perimortem}

Si bien entre las causas de paro cardiorrespiratorio en el embarazo no aparece naturalmente la COVID-19, es evidente que en pacientes con ventilación mecánica prolongada y complicaciones varias derivadas de la enfermedad, así como de la propia hospitalización, esto es una posibilidad que debe considerarse para planificar un enfrentamiento apropiado. La resucitación se realiza como en las pacientes no embarazadas, pero con un útero gestante sobre el ombligo, el útero debe desplazarse hacia la izquierda para minimizar la compresión aortocava. Asumiendo que esta emergencia ocurriría en pacientes hospitalizadas en $\mathrm{UCl}$, las vías venosas y aéreas estarán ya adecuadamente permeabilizadas. Las compresiones torácicas deben ser con la mano más cefálica que en las mujeres no embarazadas, y ciertos medicamentos necesarios pueden ser administrados por el tubo endotraqueal.

Si el útero está a más de cuatro dedos sobre el ombligo o si se conoce la edad gestacional y está en torno a las 26 semanas, o la estimación del peso fetal es $\geq 1000 \mathrm{~g}$, el feto debe extraerse a los 4 minutos de maniobras infructuosas (cesárea perimortem). Es posible que la extracción fetal favorezca en estos casos la sobrevida materna, pero el resultado neonatal sin secuelas neurológicas mejora significativamente cuando el parto ocurre dentro de los 5 minutos de ocurrido el paro cardiorrespiratorio ${ }^{33}$.

En la figura 2 presentamos una propuesta de manejo basada en la evidencia actualmente disponible, teniendo siempre en consideración que cada medida debe ser individualizada al caso particular; muchas deben ser adaptadas a las realidades de cada institución, y se debe hacer énfasis en la necesidad de un enfoque multidisciplinario.

\section{Conclusiones}

Comunicamos dos casos de gestantes con COVID19 grave durante el segundo trimestre tardío de embarazo, lo que supone un desafío a la práctica médica y al conocimiento actual de la enfermedad. A partir de ellos presentamos una propuesta de manejo basada en la evidencia disponible y hacemos hincapié en la importancia de un abordaje multidisciplinario que fomente la comunicación entre los especialistas involucrados. Finalmente, destacamos la importancia de tener en consideración que el conocimiento sobre esta enfermedad es aún escaso y aumenta día a día, por lo que se hace necesario adecuar las conductas a medida que vamos aprendiendo sobre ella.

\section{Agradecimientos}

Agradecemos al Dr. Cristián Garrido, médico del servicio de urgencias de la Clínica Universidad de los Andes y profesor de la Facultad de Medicina de la Universidad de los Andes, quien ha tenido un papel fundamental en la adaptación y la implementación clínica del colchón para pronación vigil.

\section{Financiación}

Los autores declaran no haber recibido financiación.

\section{Conflicto de intereses}

Los autores declaran no tener conflictos de intereses.

\section{Responsabilidades éticas}

Protección de personas y animales. Los autores declaran que para esta investigación no se han realizado experimentos en seres humanos ni en animales.

Confidencialidad de los datos. Los autores declaran que han seguido los protocolos de su centro de trabajo sobre la publicación de datos de pacientes. 
Derecho a la privacidad y consentimiento informado. Los autores han obtenido el consentimiento informado de los pacientes y/o sujetos referidos en el artículo. Este documento obra en poder del autor de correspondencia.

\section{Bibliografía}

1. Rasmussen SA, Jamieson DJ. Coronavirus disease 2019 (COVID-19) and pregnancy: responding to a rapidly evolving situation. Obstet Gynecol. 2020:135:999-1002.

2. Lam CM, Wong SF, Leung TN, Chow KM, Yu WC, Wong TY, et al. A case-controlled study comparing clinical course and outcomes of pregnant and non-pregnant women with severe acute respiratory syndrome. BJOG. 2004;111:771-4

3. Huntley BJF, Huntley ES, Di Mascio D, Chen T, Berghella V, Chauhan SP. Rates of maternal and perinatal mortality and vertical transmission in pregnancies complicated by severe acute respiratory syndrome coronavirus 2 (SARSCo-V-2) infection: a systematic review. Obstet Gynecol. 2020;136:303-12.

4. Blitz MJ, Grünebaum A, Tekbali A, Bornstein E, Rochelson B, Nimaroff M, et al. Intensive care unit admissions for pregnant and nonpregnant women with coronavirus disease 2019. Am J Obstet Gynecol. 2020;223:290-1.

5. Romagano MP, Guerrero K, Spillane N, Kayaalp E, Smilen SW, Álvarez M, et al. Perinatal outcomes in critically ill pregnant women with coronavirus disease 2019. Am J Obstet Gynecol MFM. 2020;2(Suppl):1-6.

6. Blitz MJ, Rochelson B, Minkoff $H$, Meirowitz N, Prasannan L, London V et al. Maternal mortality among women with coronavirus disease 2019 admitted to the intensive care unit. Am J Obstet Gynecol. 2020;223:595-599. e5.

7. Kayem G, Lecarpentier E, Deruelle P, Bretelle F, Azria E, Blanc J, et al. A snapshot of the Covid-19 pandemic among pregnant women in France. J Gynecol Obstet Hum Reprod. 2020;49:101826.

8. Guan WJ, Liang WH, Zhao Y, Liang HR, Chen ZS, Li YM, et al. Comorbidity and its impact on 1590 patients with COVID-19 in China: a nationwide analysis. Eur Respir J. 2020;55:2000547.

9. Guan WJ, Ni ZY, Hu Y, Liang WH, Ou CQ, He JX, et al. Clinical characteristics of coronavirus disease 2019 in China. N Engl J Med. 2020;382:1708-20.

10. Richardson S, Hirsch JS, Narasimhan M, Crawford JM, McGinn T, Davidson $\mathrm{KW}$, et al. Presenting characteristics, comorbidities, and outcomes among 5700 patients hospitalized with COVID-19 in the New York City area. JAMA. 2020;323:2052-9.

11. Knight M, Bunch K, Vousden N, Morris E, Simpson N, Gale C, et al. Characteristics and outcomes of pregnant women admitted to hospital with confirmed SARS-CoV-2 infection in UK: national population based cohort study. BMJ. 2020;369:m2107.

12. Ellington S, Strid P, Tong VT, Woodworth K, Galang RR, Zambrano LD, et al. Characteristics of women of reproductive age with laboratory-confirmed SARS-CoV-2 infection by pregnancy status - United States, January 22-June 7, 2020. MMWR Morb Mortal Wkly Rep 2020;69:769-75.

13. Schwaiberger D, Karcz M, Menk M, Papadakos PJ, Dantoni SE. Respiratory failure and mechanical ventilation in the pregnant patient. Crit Care Clin. 2016;32:85-95.

14. Ministerio de Salud, Chile. Orientaciones para el manejo de casos SARS COV-2 (COVID-19) en gestantes, puérperas y/o díadas. Junio 2020.
15. Vega M, Hughes F, Bernstein PS, Goffman D, Sheen JJ, Aubey JJ, et al. From the trenches: inpatient management of coronavirus disease 2019 in pregnancy. Am J Obstet Gynecol MFM. 2020;2:100154.

16. Alhazzani W, Møller MH, Arabi YM, Loeb M, Gong MN, Fan E, et al. Surviving Sepsis Campaign: guidelines on the management of critically ill adults with coronavirus disease 2019 (COVID-19). Intensive Care Med. 2020;46:854-87.

17. Sun $Q$, Qiu H, Huang M, Yang Y. Lower mortality of COVID-19 by early recognition and intervention: experience from Jiangsu Province. Ann Intensive Care. 2020;10:33.

18. Guérin $C$, Reignier J, Richard JC, Beuret $P$, Gacouin A, Boulain T, et al Prone positioning in severe acute respiratory distress syndrome. N Engl J Med. 2013;368:2159-68.

19. Samanta S, Samanta S, Wig J, Baronia AK. How safe is the prone position in acute respiratory distress syndrome at late pregnancy? Am J Emerg Med. 2014;32:687.e1-6873.

20. Pacheco LD, Saad AF, Saade G. Early acute respiratory support for pregnant patients with coronavirus disease 2019 (COVID-19) infection. Obstet Gynecol. 2020;136:42-5.

21. Lucarelli E, Behn C, Lashley S, Smok D, Benito C, Oyelese Y. Mechanical ventilation in pregnancy due to COVID-19: a cohort of three cases. Am J Perinatol. 2020;37:1066-9.

22. Lapinsky SE. Management of acute respiratory failure in pregnancy. Semin Respir Crit Care Med. 2017;38:201-7.

23. Genevieve Juillet, Research and Innovation Department, University of Oxford. Low-cost dexamethasone reduces death by up to one third in hospitalised patients with severe respiratory complications of COVID-19. (Consultado el 10 de julio de 2020.) Disponible en: https://www.ox.ac.uk/ news/2020-06-16-low-cost-dexamethasone-reduces-death-one-third-hospitalised-patients-severe.

24. COVID-19 Treatment Guidelines Panel. Coronavirus disease 2019 (COVID-19) treatment guidelines. National Institutes of Health. (Consultado el 10 de julio de 2020.) Disponible en: https://www.covid19treatmentguidelines.nih.gov/.

25. Effect of antenatal dexamethasone administration on the prevention of respiratory distress syndrome. Am J Obstet Gynecol. 1981;141:276-87.

26. Roberts D, Brown J, Medley N, Dalziel SR. Antenatal corticosteroids for accelerating fetal lung maturation for women at risk of preterm birth. Cochrane Database Syst Rev. 2017;(3):CD004454.

27. Martinelli I, Ferrazzi E, Ciavarella A, Erra R, lurlaro E, Ossola M, et al. Pulmonary embolism in a young pregnant woman with COVID-19. Thromb Res. 2020;191:36-7.

28. Bilaloglu S, Aphinyanaphongs $\mathrm{Y}$, Jones S, Iturrate $\mathrm{E}$, Hochman J, Berger JS. Thrombosis in hospitalized patients with COVID-19 in a New York City Health System. JAMA. 2020;324:799-801.

29. Di Renzo GC, Giardina I. Coronavirus disease 2019 in pregnancy: consider thromboembolic disorders and thromboprophylaxis. Am J Obstet Gynecol. 2020;223:135.

30. Schnettler WT, Al Ahwel Y, Suhag A. Severe acute respiratory distress syndrome in coronavirus disease 2019-infected pregnancy: obstetric and intensive care considerations. Am J Obstet Gynecol MFM. 2020;2: 100120

31. Slayton-Milam S, Sheffels S, Chan D, Alkinj B. Induction of labor in an intubated patient with coronavirus disease 2019 (COVID-19). Obstet Gynecol. 2020;136:962-4.

32. Stefanovic V. COVID-19 infection during pregnancy: fetus as a patient deserves more attention. J Perinat Med. 2020;48:438-40.

33. Oyarzún E, Kusanovic J. Urgencias en obstetricia. Rev Med Clin Condes. 2011;22:316-31. 